\title{
Entropy generation of a hybrid nanofluid on MHD mixed convection is a lid-driven cavity with partial heating having two rounded corners
}

\author{
Zakaria Korei $^{1}$, Smail Benissaad ${ }^{1^{*}}$ \\ ${ }^{1}$ Applied energetic and pollution laboratory. Department of Mechanical Engineering, University of Mentouri Brothers Constantine 1. \\ Constantine, Algeria.
}

\begin{abstract}
This research aims to investigate thermal and flow behaviors and entropy generation of magnetohydrodynamic $\mathrm{Al} 2 \mathrm{O} 3-\mathrm{Cu} /$ water hybrid nanofluid in a lid-driven cavity having two rounded corners. A solver based on $\mathrm{C}++$ object-oriented language was developed where the finite volume was used. Parameter's analysis is provided by varying Reynolds numbers (Re), Hartmann numbers (Ha), the volume fraction of hybrid nanofluid $(\phi)$, radii of the rounded corners. The findings show that reducing the radii of the rounded corners minimizes the irreversibility. Furthermore, the thermal conductivity and dynamic viscosity of hybrid nanofluid contribute to increasing the irreversibility. Finally, the entropy generation is decreased by increasing the Hartman number and increases by rising the Reynolds number.
\end{abstract}

Keywords. Entropy. Nanofluid. Mixed convection. MHD. Lid-driven cavity. Corners.

\section{INTRODUCTION}

In most practical applications, the driving force of convection is generally a combination of buoyancy and various applied forces, resulting in mixed convection. The presence of an external electromagnetic in the latter is an essential physical phenomenon with a wide variety of industrial applications such as cooling of electronic devices, nuclear reactor, etc. Many studies are available in the literature in different configurations with and without a magnetic field.

Öztop et al. [1] investigated mixed convection in a liddriven cavity having a wavy bottom wall using nanofluid in the presence of a magnetic field. They observed that increasing the $\phi$ can lead to an improvement or reduction in heat transfer based on both numbers Ha and Ri. Khorasanizadeh et al. [2] performed mixed convection in an enclosure filled with nanofluid (Cu-water). They found that the $\mathrm{Sg}$ takes the maximum value at low $\mathrm{Ra}$ and high $\mathrm{Re}$ and minimum value when both numbers are low. Barnoona et al. [3] conducted a study about MHD mixed convection in an enclosure containing rotating cylinders filled with nanofluid using a two-phase approach. Selimefendigil et al. [4] analyzed mixed convection in an enclosure using nanofluid where the domain divided diagonally, each one affected by inclined magnetic fields. They observed that increasing $\mathrm{Ha}$ and Ri decrease the Sg. Mehrez et al. [5] investigated heat transfer in an open cavity having backward-facing step using nanofluid. They observed that increasing $\mathrm{Ri}$,
Re and $\phi$ increase the heat transfer and Sg. Berrahil et al. [6] examined MHD natural convection in an annular enclosure filled with A12O3/water nanofluid. They used variable properties to estimate the dynamic viscosity and the thermal conductivity of nanofluid; they studied the effect of serval parameters also proposed new correlations of Nusselt number (inner cylinder) for the axial and radial magnetic fields cases. Cho [7] analyzed MHD mixed convection in wavy-wall lid-driven cavity. Hussain et al. [8] examined the inclined magnetic field effects in a double lid-driven cavity with discrete heating filled with a nanofluid. Sourtiji et al. [9] studied mixed convection in a ventilated cavity using Al2O3water nanofluid. They observed that the $\mathrm{Nu}_{\mathrm{m}}$ increases when $\mathrm{Re}, \mathrm{Ri}$ and $\phi$ is increased. Thermal radiation and free convection heat transfer mechanisms of nanofluid inside a shallow cavity by lattice Boltzmann method was investigated by Safaei et al. [10].

MHD mixed convection of hybrid nanofluid in a horizontal channel containing an adiabatic obstacle was performed by Hussain et al. [11]. They observed that an increase in the Ri leads to an increase in the $\mathrm{Nu}_{\mathrm{m}}$ and $\mathrm{Sg}$. Kalidasan et al. [12] analyzed natural convection in an enclosure filled with a hybrid nanofluid containing a thermally conducting block. The conjugate natural convection of a hybrid nanofluid was numerically analyzed in a porous square enclosure using the thermal non-equilibrium model by Ghalambaz et al. [13]. Cimpean et al. [14] examined a numerical study of

\footnotetext{
* Corresponding author: benissaad.smail@umc.edu.dz
} 
hybrid nanofluid on mixed convection in a porous trapezoidal chamber.

The aim of this report is to investigate thermal and flow behaviors and entropy generation in a lid-driven cavity having two rounded corners of magnetohydrodynamic A12O3-Cu/water hybrid nanofluid. Knowing that the rounded form has various uses, such as a heat exchanger or pipeline. etc. [15]. The effects of the radii of the rounded corners, volume fraction of hybrid nanofluid, $\mathrm{Ha}$ and Re are studied.

\section{PROBLEM FORMULATION GOVERNING EQUATIONS}

Figure 1 shows the schematic diagram of the system under examination. It has a square side L, partly heated, lid-driven cavity with two rounded corners. The top wall is the $\mathrm{x}$-direction wall and is maintained at a constant temperature $(\mathrm{Tc})$. The base wall is partly heated by the wall (Th). The other walls were adiabatically taken.

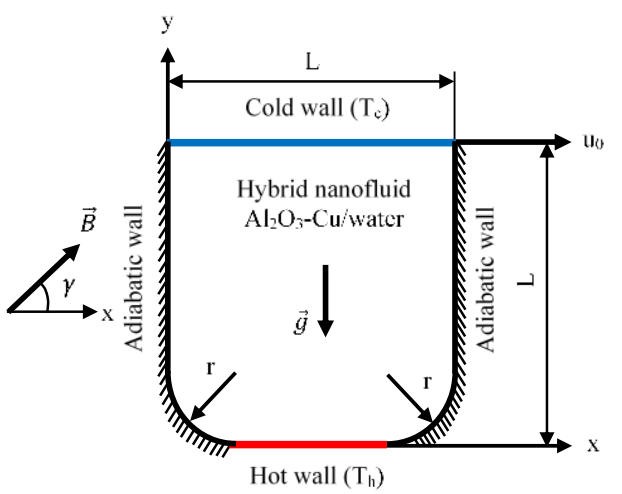

Figure 1

Problem geometry

The flow is $2 \mathrm{~d}$ laminar flow steady state, Newtonian and incompressible. The buoyancy force is calculated using Boussinesq approximations. The magnetic Reynolds number is considered small $\left(R e_{m}=\mu_{f} \sigma_{f} u_{m} L \ll 1\right)$. Buoyancy force and the Lorentz force are defined as:

$F_{\text {Buoyancy }}=(\beta \rho)_{h n f}\left(T-T_{c}\right) g$

$F_{\text {Lorenzt }}=J \times B=\sigma_{\text {hnf }} \cdot(\vec{u} \times B) \times B$

Where $J=\sigma_{\text {hnf }} \cdot(\vec{u} \times B)$

the dimensionless variables to obtain the dimensionless form of the governing equations:

$X=\frac{x}{L}, Y=\frac{y}{L}, U=\frac{u}{u_{0}}, V=\frac{v}{u_{0}}, P=\frac{p}{\rho_{f} u_{0}^{2}}, \theta=$ $\frac{T-T_{C}}{T_{h}-T_{C}}, S=s \frac{T_{0}^{2} L^{2}}{K_{f}\left(T_{h}-T_{C}\right)^{2}}$

The developed form of the dimensionless governing equations conservation of mass, momentum and energy for MHD mixed convection based on the previous hypothesis and the projections of the Lorentz force on $\mathrm{X}$ and $\mathrm{Y}$ are as follows:

$\frac{\partial U}{\partial X}+\frac{\partial V}{\partial Y}=0$
$U \frac{\partial \mathrm{U}}{\partial \mathrm{X}}+V \frac{\partial \mathrm{U}}{\partial \mathrm{Y}}=-\frac{\rho_{f}}{\rho_{h n f}} \frac{\partial \mathrm{P}}{\partial \mathrm{X}}+\frac{1}{R e} \frac{\rho_{f}}{\rho_{h n f}} \frac{\mu_{h n f}}{\mu_{f}}\left(\frac{\partial^{2} U}{\partial X^{2}}+\frac{\partial^{2} U}{\partial Y^{2}}\right)+$

$\frac{\rho_{f}}{\rho_{h n f}} \frac{\sigma_{h n f}}{\sigma_{f}} \frac{H a^{2}}{R e}\left(V \sin \gamma \cos \gamma-U \sin ^{2} \gamma\right)$

$U \frac{\partial \mathrm{V}}{\partial \mathrm{X}}+V \frac{\partial \mathrm{V}}{\partial \mathrm{Y}}=-\frac{\rho_{f}}{\rho_{h n f}} \frac{\partial \mathrm{P}}{\partial \mathrm{Y}}+\frac{1}{R e} \frac{\rho_{f}}{\rho_{h n f}} \frac{\mu_{h n f}}{\mu_{f}}\left(\frac{\partial^{2} V}{\partial X^{2}}+\frac{\partial^{2} V}{\partial Y^{2}}\right)+$ $\frac{\rho_{f}}{\rho_{h n f}} \frac{\sigma_{h n f}}{\sigma_{f}} \frac{H a^{2}}{R e}\left(U \sin \gamma \cos \gamma-V \cos ^{2} \gamma\right)+$

$\left(\frac{(\rho \beta)_{h n f}}{\rho_{h n f} \beta_{f}}\right) \operatorname{Ri\theta }$

$U \frac{\partial \theta}{\partial \mathrm{X}}+V \frac{\partial \theta}{\partial \mathrm{Y}}=\frac{\alpha_{h n f}}{\alpha_{f}} \frac{1}{\operatorname{RePr}}\left(\frac{\partial^{2} \theta}{\partial X^{2}}+\frac{\partial^{2} \theta}{\partial Y^{2}}\right)$

Dimensionless local Entropy generation:

$S_{g}=\frac{K_{h n f}}{K_{f}}\left[\left(\frac{\partial \theta}{\partial X}\right)^{2}+\left(\frac{\partial \theta}{\partial Y}\right)^{2}\right]+\chi \frac{\mu_{h n f}}{\mu_{f}}\left[2\left(\left(\frac{\partial U}{\partial X}\right)^{2}+\right.\right.$

$\left.\left.\left(\frac{\partial V}{\partial Y}\right)^{2}\right)+\left(\frac{\partial U}{\partial Y}+\frac{\partial V}{\partial X}\right)^{2}\right]+\chi H a^{2} \frac{\sigma_{h n f}}{\sigma_{f}}(U \sin \gamma-$

$V \cos \gamma)^{2}$

The appropriate boundary conditions used in this study are represented in table 1 .

Table 1

Boundary conditions

\begin{tabular}{ccccc}
\hline Cold wall & $\boldsymbol{\theta}$ & $\mathbf{P}$ & $\mathbf{U}$ & $\mathbf{V}$ \\
\hline Hot wall & 0 & $\begin{array}{c}\text { Zero } \\
\text { Gradient }\end{array}$ & 1 & 0 \\
\hline $\begin{array}{c}\text { adiabatic } \\
\text { wall }\end{array}$ & $\begin{array}{c}\text { Zero } \\
\text { Gradient }\end{array}$ & $\begin{array}{c}\text { Zero } \\
\text { Gradient }\end{array}$ & No Slip & No Slip \\
\hline
\end{tabular}

\section{NUMERICAL METHODS AND CODE VALIDATION}

A structured grid has been used in the present work, ensuring numerical solutions' stability. A solver based on the finite volume method was developed. The SIMPLE algorithm was used. The residue is selected at $1 \mathrm{e}-6$.

The grid independence study is carried out, five different grids are tested to check that the results are independent of the grid size (The full paper contains all of the details).

The validation of the numerical code was accomplished by comparing our numerical results with previously published data (The full paper contains all of the details).

\section{RESULTS AND DISCUSSION}

In this section, we present the effect of different parameters: radii of the cavity's corners, volume fraction, Hartmann number and Reynolds number; on streamlines, isotherms, $\mathrm{Nu}_{\mathrm{m}}$ and $\mathrm{Sg}$. We noticed that in this present work, the orientation of the magnetic field is in $\mathrm{X}$-direction. 
The impacts of the radii of cavity corners are shown in figure 2, which displays the streamlines (on the left), isotherms (on the right), $\mathrm{Nu}_{\mathrm{m}}$ and $\mathrm{S}_{\mathrm{g}}$ (on the middle) at $\mathrm{Ha}=50, \mathrm{Ri}=10, \mathrm{Re}=100, \phi=0.04$. This figure shows that reducing the radii of the corners increases the intensity of the clockwise circulation cell, and beside the corners, the isotherms spread towards the right walls due to an increase in convective transport. Less value of the corners' radii gave the highest $\mathrm{Nu}_{\mathrm{m}}$ and minimum $\mathrm{S}_{\mathrm{g}}$, and this is because of greater buoyancy forces to be generated within the cavity when we decrease the radii.
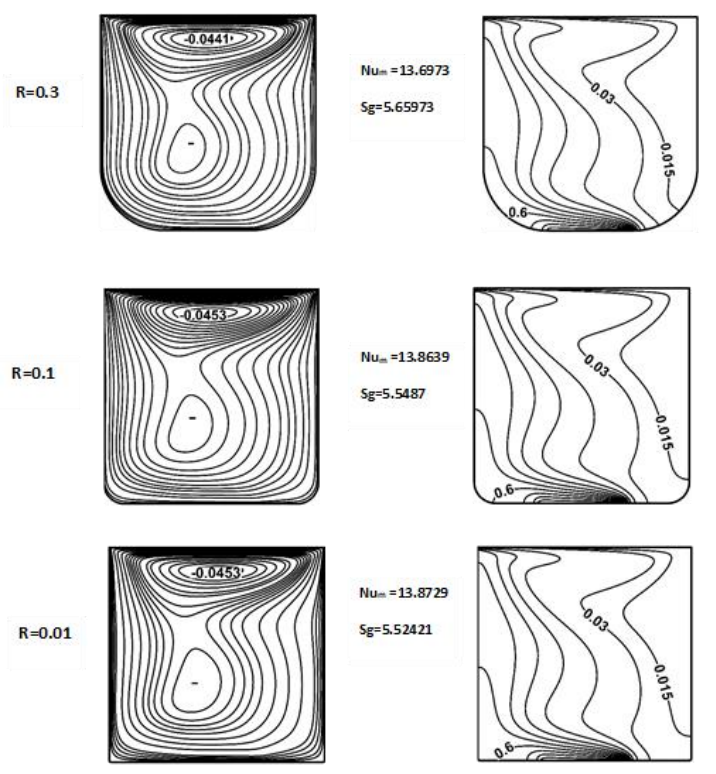

Figure 2

Streamlines (on the left), isotherms (on the right), $\mathrm{Nu}_{\mathrm{m}}$ and $\mathrm{Sg}$ (on the middle) for various radii at $\mathrm{Ha}=50$, $\mathrm{Ri}=10, \mathrm{Re}=100, \phi=0.04$.
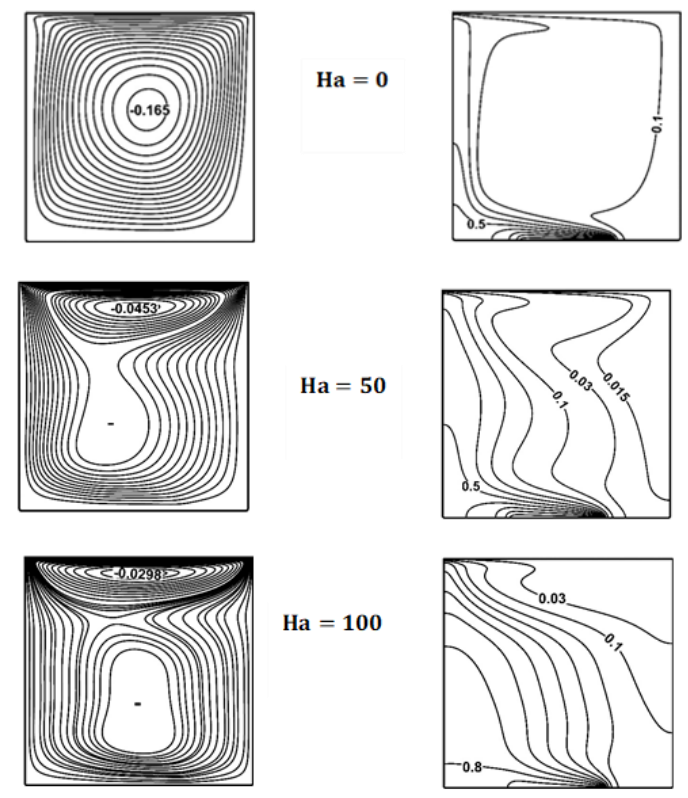

Figure 3

Streamlines (on the left), isotherms (on the right), for various $\mathrm{Ha}$ at $\mathrm{Ri}=10, \mathrm{Re}=100, \phi=0.04$

$\mathrm{R}=0.01$.
The effects of Ha on streamlines (on the left), isotherms (on the right), are presented in figure 3 at $\mathrm{Ri}=10$, $\mathrm{Re}=100, \phi=0.04$. For $\mathrm{Ha}=0$, convection-driven circulation is obvious within the cavity. But rising Ha, the main vortex rises upwards and diminishes. The sub vortex appears in the center and becomes larger. The density of isotherms declines as Ha rises and travels from left to right wall, which means that the convection flux at the higher Ha is less, damping the flow.

The variations of $\mathrm{Nu}_{\mathrm{m}}$ and $\mathrm{S}_{\mathrm{g}}$ as a function of volume fraction of hybrid nanofluid at $\mathrm{Re}=100, \mathrm{Ri}=10, \phi=$ $0.04, \mathrm{R}=0.01$ for various Hartmann numbers are presented in figure 4 . We observe that for $\mathrm{Ha}=0,25,50$, 75 increasing volume fraction increases the $S_{g}$, which is due to the enhancement of the thermal conductivity of hybrid nanofluid. However, for $\mathrm{Ha}=100$, we notice a reduction in the irreversibility.

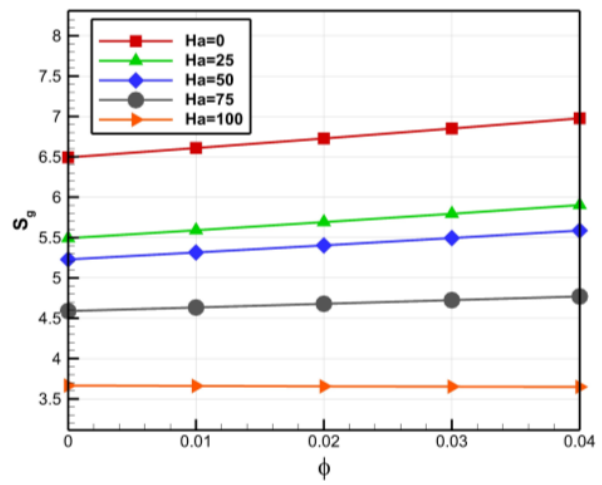

Figure 4

Variation of $\mathrm{Sg}$ with $\phi$ for different $\mathrm{Ha}$ at $\mathrm{Re}=100$, $\mathrm{Ri}=10, \phi=0.04, \mathrm{R}=0.01$.

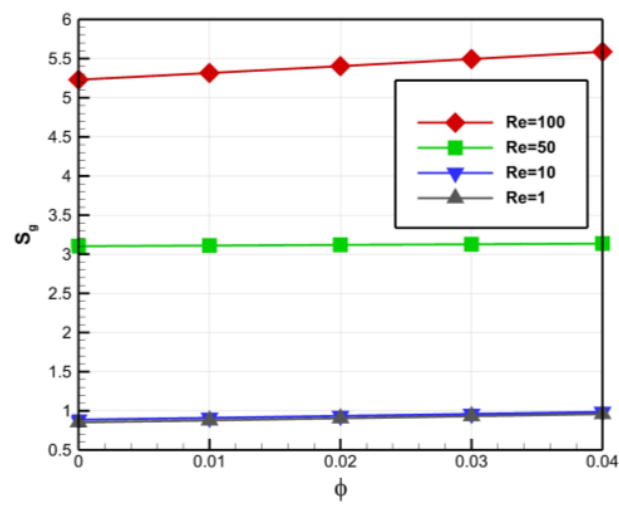

Figure 5

Variation of $\mathrm{Sg}$ with $\phi$ for different $\mathrm{Re}$ at $\mathrm{Ha}=50$, $\mathrm{Ri}=10, \mathrm{R}=0.01$.

Finally, the variation of $\mathrm{Sg}$ with $\phi$ for different $\mathrm{Re}$ at $\mathrm{Ha}=50, \mathrm{Ri}=10, \mathrm{R}=0.01$ is illustrated in figure 5. The results show that for all cases, increasing Re the average entropy generation increase. Similar behavior was observed by S. Hussain et al. [11]. The increase of 
Reynolds number leads to an increase in the velocity and temperature gradient, where these two cause this rise.

\section{Conclusions}

Entropy generation of a hybrid nanofluid on mixed convection in a lid-driven cavity having two rounded corners in the presence of a magnetic field is studied numerically using a solver based on $\mathrm{C}++$ object-oriented language. The finite volume method was used to solve governing equations. The obtained results allowed us to observe that reducing the radii of the rounded cavities' corners improves the heat transfer rate and minimizes the $\mathrm{S}_{\mathrm{g}}$. Thermal conductivity and dynamic viscosity of hybrid nanofluid contribute to increasing the irreversibility. The entropy generation decrease with the growth of the Hartmann number and rises with the increase of Reynolds number.

\section{NOMENCLATURE}

B magnetic field (T)

$g$ gravitational acceleration $\left(\mathrm{m} / \mathrm{s}^{2}\right)$

$\mathrm{Ha}$ Hartmann number $=B_{0} L \sqrt{\frac{\sigma_{f}}{\mu_{f}}}$

$K \quad$ heat conductivity ( $\mathrm{W} \mathrm{m}^{-1} \mathrm{k}^{-1}$ )

$L \quad$ cavity length (m)

$N u_{m} \quad$ Nusselt number (mean)

$\mathrm{P} \quad$ dimensionless pressure

Pr Prandtl number $=\frac{\nu_{f}}{\alpha_{f}}$

$T \quad$ temperature (K)

$\mathrm{R}$ dimensionless corner radii

$R a \quad$ Rayleigh number $=\frac{g \beta_{f}\left(T_{h}-T_{c}\right) L^{3}}{v_{f} \alpha_{f}}$

Re Reynolds number $=\frac{\rho_{f} u_{0} L}{\mu_{f}}$

$R i \quad$ Richardson number $=\frac{g \beta_{f}\left(T_{h}-T_{c}\right) L}{V^{2}}$

$\mathrm{S}_{\mathrm{g}} \quad$ Average dimensionless entropy

$\mathrm{X}, \mathrm{Y}$ dimensionless coordinates

$\mathrm{U}, \mathrm{V}$ dimensionless velocity components

\section{Greek symbols}

$v \quad$ kinematic viscosity $\left(\mathrm{m}^{2} \mathrm{~s}^{-1}\right)$

$\mu \quad$ dynamic viscosity $\left(\mathrm{kg} \mathrm{m}^{-1} \mathrm{~s}^{-1}\right)$

$\alpha \quad$ thermal diffusivity $\left(\mathrm{m}^{2} \mathrm{~s}^{-1}\right)$

$\beta \quad$ thermal expansion coefficient $\left(\mathrm{K}^{-1}\right)$

$\sigma \quad$ electrical conductivity $\left(\Omega^{-1} \mathrm{~m}^{-1}\right)$

$\mathrm{J} \quad$ current density $\left(\mathrm{A} / \mathrm{m}^{2}\right)$

$\rho \quad$ density $\left(\mathrm{kg} / \mathrm{m}^{3}\right)$

$\gamma \quad$ magnetic field angle

$\theta \quad$ dimensionless temperature

$\chi \quad$ irreversibility factor $=\frac{\mu_{f} T_{0}}{k_{f}}\left(\frac{u_{0}}{T_{h}-T_{c}}\right)^{2}$

$\phi \quad$ nanoparticles volume fraction

\begin{tabular}{ll}
\multicolumn{2}{l}{ Subscripts } \\
$\mathrm{m}$ & Mean variable \\
hnf & Hybrid nanofluid \\
$\mathrm{f}$ & fluid \\
$\mathrm{c}$ & cold \\
$\mathrm{h}$ & hot
\end{tabular}

1. H.F. Öztop, A. Sakhrieh, E. Abu-Nada, K. AlSalem, Mixed convection of MHD flow in NANOFLUID filled and partially heated wavy walled lid-driven enclosure, International Communications in Heat and Mass Transfer. 86 (2017) 42-51.

2. H. Khorasanizadeh, M. Nikfar, J. Amani, Entropy generation $\mathrm{OF} \quad \mathrm{Cu}$-water NANOFLUID mixed convection in a cavity, European Journal of Mechanics - B/Fluids. 37 (2013) 143-152.

3. P. Barnoon, D. Toghraie, R.B. Dehkordi, H. Abed, MHD mixed convection and entropy generation in A lid-driven cavity with rotating cylinders filled by a nanofluid using two PHASE mixture model, Journal of Magnetism and Magnetic Materials. 483 (2019) 224-248.

4. F. Selimefendigil, H.F. Öztop, A.J. Chamkha, Mhd mixed convection and entropy generation of nanofluid filled lid driven cavity under the influence of inclined magnetic fields imposed to its upper and lower diagonal triangular domains, Journal of Magnetism and Magnetic Materials. 406 (2016) 266-281.

5. Z. Mehrez, M. Bouterra, A. El Cafsi, A. Belghith, Heat transfer and entropy generation analysis of nanofluids flow in an open cavity, Computers \&amp; Fluids. 88 (2013) 363-373. doi:10.1016/j.compfluid.2013.09.026.

6. F. Berrahil, A. Filali, C. Abid, S. Benissaad, R. Bessaih, O. Matar, Numerical investigation on natural convection of al2o3/water nanofluid with variable properties in an annular enclosure under magnetic field, International Communications in Heat and Mass Transfer. 126 (2021) 105408.

7. C. C. Cho, Mixed convection heat transfer and entropy generation of cu-water nanofluid in wavy-wall lid-driven cavity in presence of inclined magnetic field, International Journal of Mechanical Sciences. 151 (2019) 703-714.

8. S. Hussain, K. Mehmood, M. Sagheer, MHD mixed convection and entropy generation of water-alumina nanofluid flow in a double lid driven cavity with discrete heating, Journal of Magnetism and Magnetic Materials. 419 (2016) 140-155. 
9. E. Sourtiji, M. Gorji-Bandpy, D.D. Ganji, S.F. Hosseinizadeh, Numerical analysis of mixed convection heat transfer of al2o3-water nanofluid in a ventilated cavity considering different positions of the outlet port, Powder Technology. 262 (2014) 71-81.

10. M.R. Safaei, A. Karimipour, A. Abdollahi, T.K. Nguyen, The investigation of thermal radiation and free convection heat transfer mechanisms of nanofluid inside a shallow cavity by lattice Boltzmann method, Physica A 509: pp 515-535, 2018.

11. S. Hussain, S.E. Ahmed, T. Akbar, Entropy generation analysis IN MHD mixed convection of hybrid Nanofluid in an Open cavity with a HORIZONTAL channel containing an ADIABATIC OBSTACLE, International Journal of Heat and Mass Transfer. 114 (2017) 1054-1066.

12. K. Kalidasan, R. Velkennedy, P. Rajesh Kanna, Laminar natural convection of copper - Titania/Water hybrid Nanofluid in an open ended C - shaped enclosure with an Isothermal block, Journal of Molecular Liquids. 246 (2017) 251-258.

13. M. Ghalambaz, M.A. Sheremet, S.A. Mehryan, F.M. Kashkooli, I. Pop, Local thermal NON-EQUILIBRIUM analysis of Conjugate FREE convection within a porous enclosure occupied With $\mathrm{Ag}-\mathrm{MgO}$ hybrid Nanofluid, Journal of Thermal Analysis and Calorimetry. 135 (2018) 1381-1398.

14. D.S. Cimpean, M.A. Sheremet, I. Pop, Mixed convection of hybrid nanofluid in a porous trapezoidal chamber, International Communications in Heat and Mass Transfer. 116 (2020) 104627.

15. Z. Korei, S. Benissaad, Turbulent forced convection and entropy analysis of a NANOFLUID through a 3D $90^{\circ}$ ELBOW using A two-phase approach, Heat Transfer. (2021). 